Design and methods We assessed BDNF levels in 27 PI (DSM $\mathrm{V}$ criteria) patients (15 males, 56\%) and in 14 controls (6 males, 43\%). All subjects underwent 18fluorodeoxyglucose positron emission tomography (18F-FDG PET), brain PET with $13 \mathrm{~N}$-ammonium in the morning in wakefulness. Brain PET/CT scanning was performed $30 \mathrm{~min}$. after administration of $250 \mathrm{MBq} 18 \mathrm{~F}-\mathrm{FDG}$. The PET images were statistically analyzed by 3D-SSP method (Z-score) using software package Cortex ID.

Results There was no difference in BDNF level in PI patients and control group: $11.9(8.5 ; 100)$ and $12.8(10.1 ; 72.8) \mathrm{ng} /$ $\mathrm{ml}$, respectively $(\mathrm{p}=0,138)$. We found a weak association between BDNF level and brain metabolism in the left prefrontal medial lobe $(\rho=0.57, \mathrm{p}=0.02)$ and right parietal inferior lobe $(\rho=0.52, \mathrm{p}=0.039)$. However, there was no association between BDNF level and $13 \mathrm{~N}$-ammonium accumulation which indicate the activity of glutamate synthase.
Conclusions Insomnia is not associated with the changes in BDNF levels compared to healthy control subjects, although the relation between brain metabolism and BDNF concentration requires further investigation.

(The study was supported by the Grant of the President of Russian Federation for the Leading Scientific Schools of Russia HШ-5508.2018.7 (agreement № 075-15-2019-161, 23.05.2019).

\section{P067 MEDICAL STUDENT EDUCATION IN SLEEP AND ITS DISORDERS: STILL MEAGRE 20 YEARS ON}

${ }^{1}$ Stephanie Romiszewski*, ${ }^{1}$ Felix May, ${ }^{1}$ Jane Homan, ${ }^{1}$ Ben Norris, ${ }^{2}$ Michelle Miller, ${ }^{3}$ Adam Zeman. ${ }^{1}$ Royal Devon and Exeter NHS Foundation Trust, Exeter, UK; ${ }^{2}$ University of Warwick, Warwick, UK; ${ }^{3}$ Exeter University, Exeter, UK

10.1136/bmjresp-2019-bssconf.67

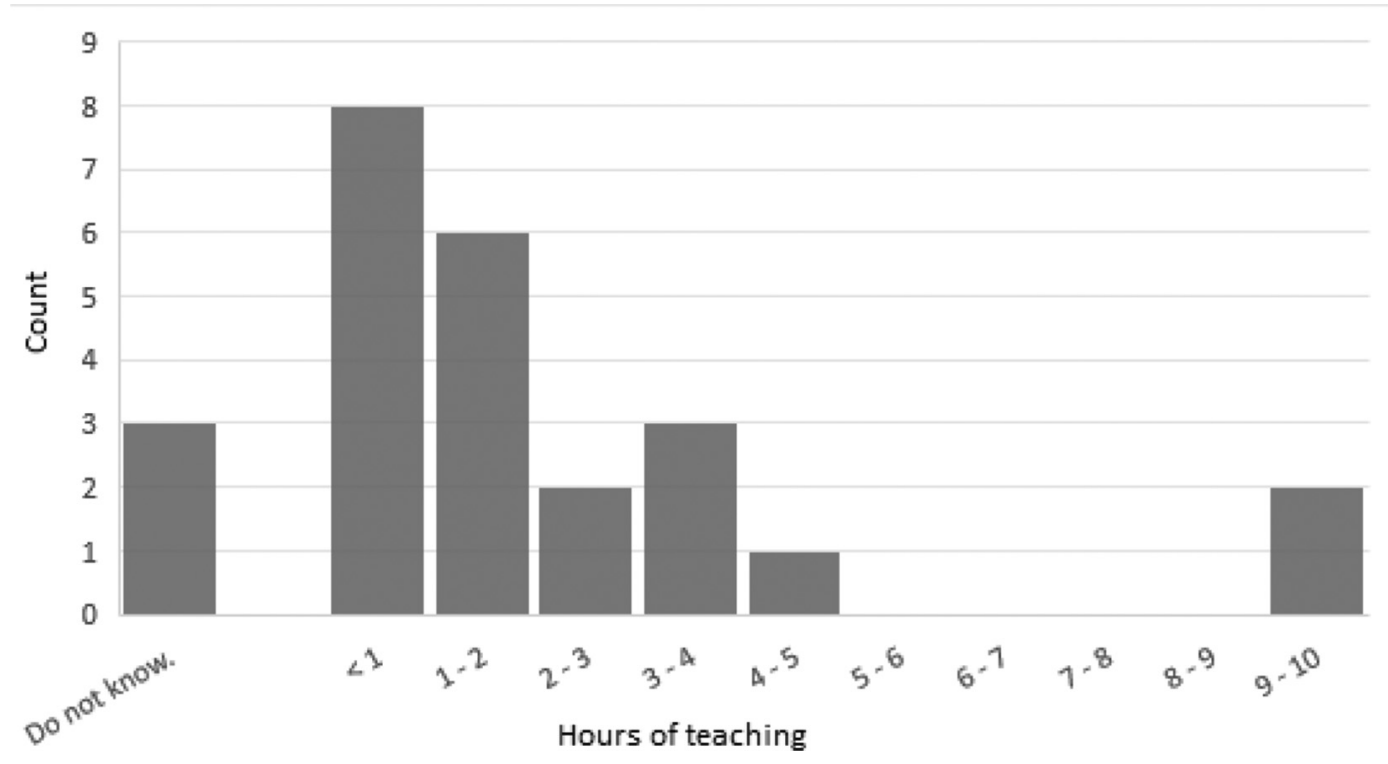

Abstract P067 Figure 1 Distribution of estimated hours of teaching time spent on sleep medicine UK medical degree courses

\begin{abstract}
Respiratory sleep conditions
Sleep disorders in psychiatry

Management of sleep disorders

Insomnia

Biology of sleep

Sleep disorders in general medicine Circadian rhythm disorder Hypersomnias (non-respiratory) Sleep diagnostics and investigations Parasomnias

Sleep disorders in children

Other sleep disorders
\end{abstract}

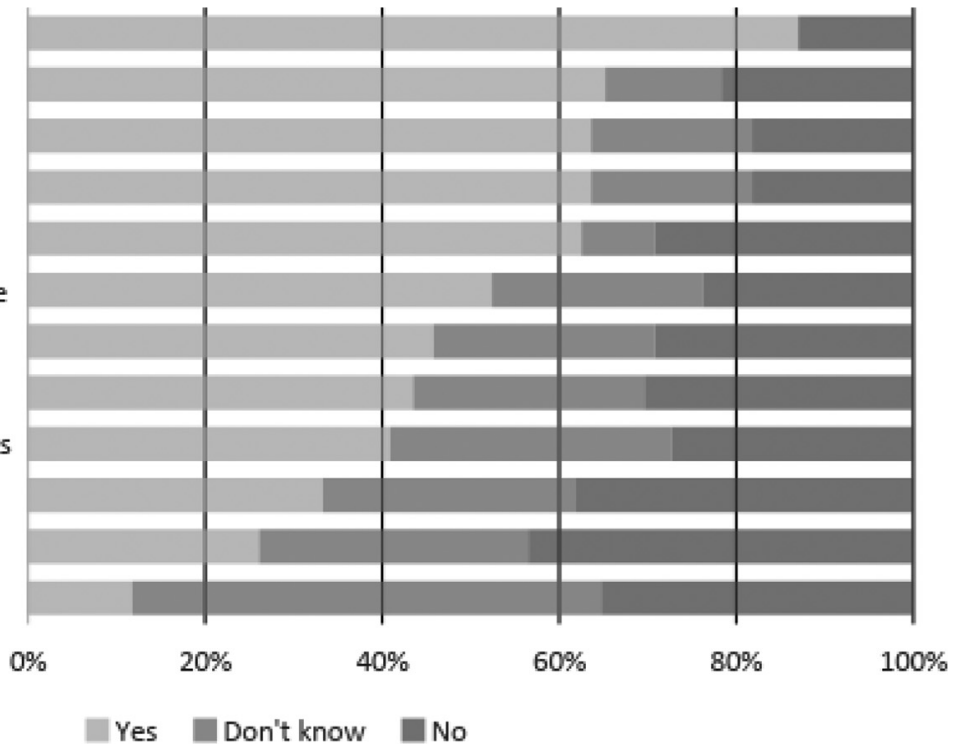

Abstract P067 Figure 2 Proportions of sleep-related topics covered in current UK medical education 
Introduction Stores' questionnaires survey in 1998 found that UK medical students received a median of 20 minutes education about sleep. We asked whether this situation has improved.

Methods A cross sectional survey of 34 medical degree courses in the UK adapted from Stores '1998 questionnaire including time spent on sleep teaching medicine, sub-topics covered, and forms of assessment.

Results 25 (74\%) UK medical schools responded to our survey. The time spent devoted to sleep medicine during undergraduate training was median 1.5 hours, mode $<1$ hour, and mean 3.2 hours (standard deviation $=2.6$, figure 1 ). Only two schools had a sleep syllabus or dedicated compulsory module ( $8 \%$, figure 2$)$. When asked whether sufficient time is allotted to sleep and its disorders, 50\% said yes, 38\% said no and $13 \%$ were unsure. Free text comments made by our respondents had recurring themes: sleep medicine is typically subsumed into teaching by other specialities, with the result that course directors are uncertain about the details of provision; obstructive sleep apnoea is often identified as the key or only relevant sleep disorder, knowledge of sleep disorders is regarded as optional, and there is inertia about the prospect of change. However, a substantial minority of respondents are enthusiastic about making improvements to the sleep education they currently provide.

Discussion Sleep medicine remains a neglected topic despite agreement on the importance of sleep in general health. Obstacles to change are similar to those noted by Stores 20 over 20 years ago, including the views that 'sleep is not a core topic' or the 'curriculum is already too crowded'. Given that doctors are often the first point of contact for the public, we recommend that medical schools should establish and implement a sleep medicine curriculum. We suggest a simple syllabus, available on request.

\section{REFERENCES}

1. Greenstone M, Hack M. Obstructive sleep apnoea. BMJ Br. Med. J 2014;348.

2. Ferrie JE, Kumari $M$, Salo $P$, Singh-Manoux $A$, Kivimäki $M$. Sleep epidemiology-A rapidly growing field. Int. J. Epidemiol 2011;40:1431-1437.

3. Bjorvatn B, Grønli J, Pallesen S. Prevalence of different parasomnias in the general population. Sleep Med 2010;11:1031-1034.

4. Hafner M, Stepanek M, Taylor J, Troxel WM, van Stolk C. Why sleep matters - the economic costs of insufficient sleep: a cross-country comparative analysis. Rand health quarterly 2017;6.

5. NICE. Continuous positive airway airway pressure for the treatment of obstructive sleep apnoea/hypopnoea syndrome. Technology Appraisal Guidance 2008.

6. Zeman A, Zaiwalla Z. Prescribing sodium oxybate for narcolepsy. BMJ 2016;353: i2367.

7. National Institute for Health and Care Excellence. Insomnia. Clinical Knowledge Summaries 2015. Available at: https://cks.nice.org.uk/insomnia. (Accessed: 3rd January 2018)

8. Stores G, Crawford C. Medical student education in sleep and its disorders. $J R$ Coll Physicians L 1998:32:149-153.

9. Rosen RC, Rosekind M, Rosevear C, Cole WE, Dement WC. Physician education in sleep and sleep disorders: A national survey of U.S. medical schools. Sleep 1993;16:249-254.

10. Mindell, J. A. et al. Sleep education in medical school curriculum: A glimpse across countries. Sleep Med 2011;12:928-931.

11. Dyas JV. et al. Patients' and clinicians' experiences of consultations in primary care for sleep problems and insomnia: A focus group study. Br. J. Gen. Pract 2010:60:180-200.

12. Nowell LS, Norris JM, White DE, Moules NJ. Thematic Analysis: Striving to Meet the Trustworthiness Criteria. Int. J. Qual. Methods 2017;16:1-13.

13. Youngren WA, Miller KE, Davis JL. An Assessment of Medical Practitioners' Knowledge of, Experience with, and Treatment Attitudes Towards Sleep Disorders and Nightmares. J. Clin. Psychol. Med. Settings 2019;26:166-172.

14. Salas RME, et al. Incorporating sleep medicine content into medical school through neuroscience core curricula. Neurology 2018;91:597-610.

15. Smith AG. A sleep medicine medical school curriculum. Neurology 2018;91:587588.

\section{P068 SLEEP QUALITY IN ATHLETES AND EXERCISERS}

Rui Pereira*, Iuliana Hartescu, Robin Jackson, Kevin Morgan. Clinical Sleep Research Unit, School of Sport, Exercise and Health Sciences, Loughborough University, Loughborough, UK

\subsection{6/bmjresp-2019-bssconf.68}

Introduction Insomnia symptoms are highly prevalent in athlete populations, with $64 \%-70 \%$ of athletes describing nonrestorative sleep, frequent awakenings at night, daytime

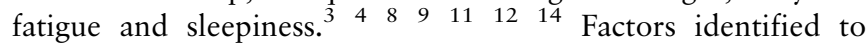
contribute to poor sleep in athletes are intense schedule of training, competition and travelling, early morning training and night-time competition. ${ }^{5} \quad 6 \quad 9 \quad 11 \quad 15$ The type of sport practiced is also a significant factor, with aesthetic sports and individual sports causing more sleep problems than team ball sports and team sports, respectively. ${ }^{13} 16$ The Cognitive Model of Insomnia explains that a transition from wake to sleep is impaired by cognitive over-activity and intrusive thoughts. ${ }^{7}$ The concept of hyperarousal has become a core component of contemporary explanatory models of insomnia. ${ }^{1} 2 \quad 7 \quad 10$ Our study aims to 1) explore the relationship between arousal, sleep vulnerability and sporting performance, and 2) to inform of what type of sports may select for higher levels of arousal and/or sleep vulnerability.

Methods The study is a controlled online survey designed to compare elite athletes and a non-competitive/lower-level competitive high exercising control group in eight different sections. The survey comprises validated questionnaires (IPAQ; PSQI; FIRST; PSAS; ESS; VAFS; 5-MEQ; TIPI; GAD-7) and additional questions designed to address topics such as napping, fatigue, training schedule and competition schedule.

Results Preliminary results (table 1) from the pilot study $(n=6)$ show the average sleep time in both groups is at recommended guidelines. Competitive elite athletes report a higher level of arousal in the PSAS cognitive scale (21 vs 17.75), higher fatigue ( 8 vs 6.5 ) and a discrepancy in obtained and desired sleep time when compared to competitive nonelite athletes.

Discussion Elite competitive athletes show a significant cognitive hyperarousal profile, which could have implications for both performance and potential sleep interventions. There are

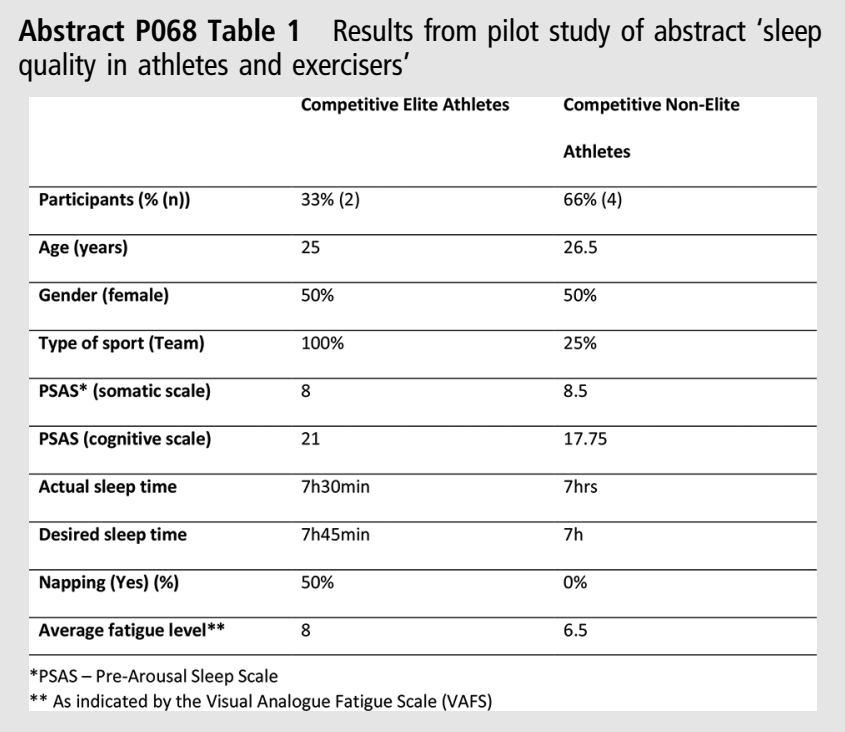

